\title{
Identification and Experimental Validation of Immune-Associate IncRNAs for Predicting Prognosis in Cervical Cancer
}

Jing $\mathrm{Ye}^{\mathrm{I}}$

Xiaojing Chen ${ }^{2}$

Weiguo Lu'

'Department of Gynecologic Oncology, Women's Hospital, Zhejiang University School of Medicine, Hangzhou, Zhejiang, People's Republic of China; ${ }^{2}$ Women's Reproductive Health Laboratory of Zhejiang Province, Women's Hospital, Zhejiang University School of Medicine, Hangzhou, Zhejiang, People's Republic of China
Correspondence: Jing Ye Department of Gynecologic Oncology, Women's Hospital, Zhejiang University School of Medicine, Hangzhou, 310000 ,

Zhejiang, People's Republic of China

$\mathrm{Tel}+86-13588756826$

Email yezi200805I8@zju.edu.cn
Purpose: Cervical cancer (CC) is a major risk for health of modern women. Immune-related long non-coding RNAs (lncRNAs) can also serve as prognostic markers of overall survival (OS) in patients with CC. This study aimed to identify an immune-related lncRNA signature for the prospective assessment of prognosis in $\mathrm{CC}$ patients.

Methods: We first calculated immune scores of CC patients in the Cancer Genome Atlas (TCGA) database. Univariate Cox, Lasso Cox and multivariate Cox regression analyses were perfumed to establish an immune-relative lncRNA signature. In addition, we processed pathway enrichment analysis and immune infiltration analysis between patients with higher or lower risk. Finally, T-cell Chemotaxis assays were processed to verify the function of 2 key lncRNAs.

Results: Our results suggested that patients with higher immune scores had longer survival time and some lncRNAs expressed differentially between two groups. Eight lncRNAs (LINC02802, LINC01877, RBAKDN, LINC02480, WWC2-AS2, LINC01281, ZBTB20AS1, IFNG-AS1) were identified as prognostic signatures for $\mathrm{CC}$. The immune-related lncRNA signature was correlated with disease progression and worse prognosis. Immune infiltration analysis indicated that the expression of 8-lncRNA signatures were corrected with infiltration level of immune cell subtypes. In addition, T-cell Chemotaxis assay validated that 2 key lncRNAs (ZBTB20-AS1 and LINC01281) could significantly promote the migration ability of $\mathrm{T}$ cells to $\mathrm{CC}$ cells.

Conclusion: Our finding demonstrated the value of lncRNAs in evaluating the immune infiltrate of the tumor. The 8-lncRNA signature could predict the prognosis of $\mathrm{CC}$ and contribute to decisions regarding the immunotherapeutic strategy.

Keywords: cervical cancer, immune-associated cell, long non-coding RNA, prognosis model, the Cancer Genome Atlas

\section{Plain Language Summary}

Cervical cancer (CC) is a major risk for health of modern women. Some studies have found that tumor immune microenvironment played an important role during the development of CC. This study aimed to identify an immune-related lncRNA signature for the prospective assessment of prognosis in CC patients. A lncRNA prognostic model was constructed through bioinformatic analysis to evaluate the prognosis risk of CC based on lncRNA expression level of CC patients. In addition, a nomogram based on this prognosis model has also been established to follow on clinical diagnostic. T-cell chemotaxis assay were processed to verify the function of 2 key lncRNAs on migration ability of T cells to CC cells. The results showed that 8-lncRNA prognosis model could diagnose the survival rate of CC 
patients. The 8-lncRNA signature-based nomogram could accurately predict the survival rate of CC patients. ZBTB20-AS1 and LINC01281 could regulate the migration ability of $\mathrm{T}$ cells to $\mathrm{CC}$ cells. This study screened an 8-lncRNA model and constructed a nomogram to predict the prognosis of $\mathrm{CC}$ patients. The 8-lncRNA signature can effectively make the prognosis evaluation of CC patients.

\section{Introduction}

Cervical cancer (CC) is one of the most common cancers threatening the health of women. An estimated 13,170 women in the United States (US) will be diagnosed with invasive cervical cancer in 2019. ${ }^{1}$ Although the incidence of CC decreased in recent years, it is still the leading cause of cancer death. In the past few years, cervical screening and human papilloma virus (HPV) vaccination has significantly increased the life expectancy of potential $\mathrm{CC}$ patients. ${ }^{1-3}$ However, the patients who diagnosed $\mathrm{CC}$ at advanced stages still have no effective treatment method. Surgical treatment combined with chemotherapy, radiotherapy and targeted therapy are common treatment methods of CC. ${ }^{4}$ With the development of cellular molecular biology and immunology, immunotherapy has become a new treatment approach for cervical cancer. ${ }^{5}$ For example, Immune Checkpoint Proteins CTL4 and PD-L1 can promote the development of $\mathrm{CC}^{6,7}$ And immune check point inhibitors (anti-PD-1/anti-CTLA-4) play important roles in CC treatment. ${ }^{8}$ Therefore, the role of tumor immune microenvironment in the pathogenesis of $\mathrm{CC}$ needs to be further explored.

Tumor immune microenvironment is widely involved in tumorgenesis of CC. Some researchers found immune cells such as $\mathrm{T}$ cell, Macrophages, and Dendritic cells could affect tumorigenesis and clinical outcome of patients. $^{9-11}$ For example, a single-cell sequencing study found 17 tumor-associated macrophage phenotypes, $22 \mathrm{~T}$ cell phenotypes, and a distinct immune composition correlated with progression-free survival of clear cell renal cell carcinoma (ccRCC) patients. ${ }^{12}$ For $\mathrm{CC}$, some researchers have found that lymphocytes infiltration level in tumor tissue was related to clinical outcome. $^{13}$ At the same time, some studies on immunotherapy have been reported. For example, PD-1/PDL1 antibody was used to suppress immune escape of cancer cell. ${ }^{6}$ Drugs like KEYNOTE-158 have also shown promising results in clinical trials. ${ }^{8}$ In conclusion, it is necessary to further explore tumor immune microenvironment in $\mathrm{CC}$ patients.
Long non-coding RNAs (LncRNAs) play critical roles in CC development. ${ }^{14}$ Various of IncRNAs were found that they could affect the development of CC. For example, LncRNA CRNDE was identified to play an oncogenic role by regulating the expression of CCNB1. ${ }^{14}$ Expression level of lncRNA ZFAS1 was associated with chemotherapy therapeutic effect. ${ }^{15}$ LncRNAs also play important roles in regulating immune response. LncRNA NKLA could promote tumor immune evasion by inhibiting T cell activation. ${ }^{16}$ LncRNA SATB2-AS1 could modulate tumor microenvironment and inhibit tumor metastasis. ${ }^{17}$ In this study, we aimed to estimate immune infiltration landscape and screen prognostic lncRNA signature in patients with CC.

\section{Materials and Methods Data Downloading}

We downloaded clinical follow-up data and gene expression data from the Cancer Genome Atlas (TCGA) database. The inclusion criterion was patients who were followed up for more than 1 days, and a total of 291 patients were included in the study.

\section{Immune Score and Mechanism Score Analysis}

Immune infiltration information was obtained from tumor immune estimation resource (TIMER). TIMER database is a comprehensive resource for systematical analysis of immune infiltrates across diverse cancer types. Researchers can use TIMER database to estimate immune infiltration level in cancer. Subsequently, the $\mathrm{R}$ package ESTIMATE was employed to assess the immune cell infiltration levels including the immune score, tumor purity, and stromal score according to the software manual for each tumor sample in the TCGA-cohort. The ESTIMATE algorithm is applied to a normalized expression matrix to estimate stromal score and immune score for each CC sample.

\section{Identification of Differentially Expressed Gene (DEG)}

Transcripts were first divided to mRNA and lncRNA with human genome data GRCh38, then differential expression analysis was processed. Patients were divided into two groups by median of immune score and stromal score. Differential expression analysis was processed with $\mathrm{R}$ package edgeR. The standard of DEGs was false discovery rate (FDR) adjusted $p$-value $<0.05$ and $|\log 2 \mathrm{FC}|>1$. 


\section{Pathway Enrichment Analysis}

KEGG (Kyoto Encyclopedia of Genes and Genomes) and GO (Gene ontology) Pathway enrichment analysis were processed with $\mathrm{R}$ package ClusterProfiler. Pathway enriched with FDR adjusted p-value $<0.05$ were considered significantly enriched.

\section{Construction of Prognosis Model}

An 8-lncRNA risk model was constructed by using univariate Cox, least absolute shrinkage and selection operator (LASSO) Cox and multivariate Cox regression analysis. Briefly, patients were first divided to training cohort and validation cohort randomly by $7: 3$. Then, univariate Cox analysis was processed by $\mathrm{R}$ package survival. IncRNAs with $P<0.05$ were further processed by lasso Cox analysis with R package glmnet. Finally, lncRNA signatures were used to build prognosis model by multivariate Cox regression with $\mathrm{R}$ package survminer. The formula of prognosis model is as follows:

$$
\text { Riskscore }=\sum_{i=1}^{k}\left(\text { Coef }_{i} \times x_{i}\right)
$$

In this formula, $\operatorname{Coef}_{i}$ represents the risk factor, $x_{i}$ represents the relative expression level of each lncRNA, Risk score represents the risk score of each patient. Then, the model was verified by survival analysis and receiver operating characteristic (ROC) curve in both training and validation cohort.

\section{Gene Set Enrichment Analysis (GSEA)}

GSEA was used to research the differences among CC patients at different risk. Patients were first grouped according to median of risk score. Then, GSEA software was used to analyze the difference of pathway activity. The $P<0.05$ was considered to be significantly enriched.

\section{Correlation Analysis of IncRNA and Immune Cells}

Immune infiltration level of each patient was calculated with R package CIBERSORT. CIBERSORT algorithm was used to analyze 22 types of immune cell. Pearson correlation coefficient was used to treat the correlation between immune cell abundance. Correlation between lncRNA and immune cell abundance was also estimated by Pearson's correlation coefficient.

\section{Independence Analysis and Nomogram Construction}

Independence analysis was processed by using $\mathrm{R}$ package survival. Univariate and multivariate regression analysis were processed to validate the prognosis model. The $P<$ 0.05 were considered independent factor. Finally, a nomogram was constructed with $\mathrm{R}$ package regplot.

\section{Cell Line and Culture Conditions}

The human CC cells and human peripheral blood mononuclear cells (PBMC) were purchased from Beina Chuanglian Biotechnology Institute (BNCC, Beijing, China). The human CC cells (BNCC337633) were cultured in PRME-1640 with 10\% FBS. PBMC (BNCC341622) were cultured in RPMI-1640 with 10\% FBS and 1\% Penicillin/streptomycin (P/S). All cell lines were cultured maintained in a humidified incubator containing $5 \% \mathrm{CO}_{2}$ at $37^{\circ} \mathrm{C}$.

\section{Plasmid Transfection}

Empty vector without ZBTB20-AS1 and LINC01281 were regarded as control. Lipofectamine 3000 was used to transfect oe-ZBTB20-AS1 vector and oe- LINC01281 vector (Genepharma, Shanghai, China) and corresponding empty vector into cell lines (Thermo Fisher Scientific, USA).

\section{RNA Extraction and qRT-PCR}

Total RNA was extracted using TRIzol reagent (Invitrogen, USA). Complementary of extracted RNA was performed using PrimeScript real-time reagent kit (Takara, Japan). All qRT-PCR was performed by using the SYBR Select Master Mix (Applied Biosystems, USA). GAPDH was used as a reference. Relative expression of targeted genes was calculated using 2- ${ }^{\Delta \Delta \mathrm{Ct}}$ method. GAPDH sense: 5'-AACG GATTTGGTCGTATTGGG-3'; GAPDH antisense: 5'CGCTCCTGGAAGATGGTGAT-3'. ZBTB20-AS1 sense: 5'-CTCTGCAACAAGACTTTCACCG C-3'; ZBTB20-AS1 antisense: 5'-AGGAGAAGGAGCGCCAACAGAT-3'. LI NC01281 sense: 5'-CTGTTGCCTACCCGAGAACA-3'; LINC01281 antisense: 5'-TGCTCACAGTCCCCATTGAG -3'. CXCL10 sense: 5'-GGTGAGAAGAGATGTCTGA ATCC -3'; CXCL10 antisense: 5'-GTCCATCCT TGGAAGCAC TGCA-3'. CXCL9 sense: 5'- CTGTT CCTGCATCAGCACCAAC-3'; CXCL9 antisense: 5'ACACACTTGGCGGTTCTTTCGG -3'. CXCL11 sense: 5'AAGGAC AACGATGCCTAAATCCC-3'; CXCL11 
antisense: 5'- CAGATGCCCTTTTCCAGGACTTC-3'. Each experiment was replicated three times.

\section{T-Cell Chemotaxis Assay}

The process of this experiment was implemented as described in former study. ${ }^{18,19}$ Briefly, T cells were isolated from PBMC through immunomagnetic negative selection (STEMCELL Technologies, Canada). Isolated T cells were pre-activated by $100 \mathrm{ng} / \mathrm{mL}$ CD3 antibody (Abcam, Shanghai, China) and recombination $10 \mathrm{ng} / \mathrm{mL}$ IL-2 (Abcam, Shanghai, China). Cancer cells were transfected and cultured for $24 \mathrm{~h}$. The supernatant of cancer cell medium was collected. Polycarbonate membranes and cancer cell conditioned medium was placed in the bottom of the well. $\mathrm{T}$ cells $\left(5 \times 10^{5}\right)$ were seeded and cultured on Transwell inserts (diameter, $6.5 \mathrm{~mm}$ ) containing 5 - or $3-\mu \mathrm{m}$ pore size (Costar, USA). After $2 \mathrm{~h}$ of incubation, the Transwell inserts were lifted. The number of transitional cells was measured by a hemocytometer, cell migrated were normalized to $\mathrm{NC}$ group (cell migrated $(\%)=$ Cell migrated/Cell migrated in $\mathrm{NC} \times 100 \%$ ). Each experiment was replicated triplicates.

\section{Statistical Analysis}

Statistical analysis was conducted mainly by $\mathrm{R}$ software (CRAN, USA) and GraphPad Prism 8 (GraphPad, USA). All data were presented as mean $\pm \mathrm{SD}$. Differences among groups were analyzed by one-way analysis of variance (ANOVA) or $t$-tests (2 groups). Pearson's correlation

\section{A}

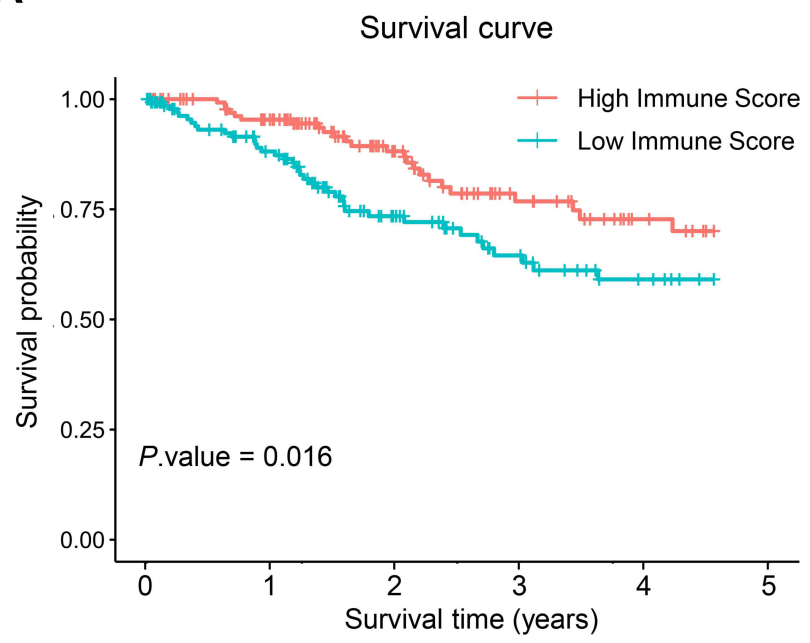

coefficient was calculated to measure the correlation. All $P$ values $<0.05$ were considered to indicate statistical significance.

\section{Results}

\section{Low Immune Scores are Associated with Poor Prognosis}

To assess the potential relationship of stromal and immune scores with patients' outcome, CC cases were categorized into high-score and low-score groups by the median expression value. Patients with a high immune score yielded better overall survival (OS) than those with a low immune score (Figure 1A). Meanwhile, higher stromal score showed no significant benefits in OS (Figure 1B).

\section{Identification of Immune-Related IncRNAs}

Since survival curve revealed a close relationship between immune score and OS. Differential gene expression analysis was processed to screen DEGs between high and low immune score groups. The results showed that 1589 mRNAs significantly up-regulated in high immune score group, while 989 mRNAs significantly down-regulated (Figure 2A). Differential gene expression analysis of lncRNA showed 290 lncRNAs significantly up-regulated in high immune score group, while 120 lncRNAs significantly downregulated (Figure 2B). A total of 2578 differential expression mRNAs (DEmRNAs) and 410 DElncRNAs were found.

\section{B}

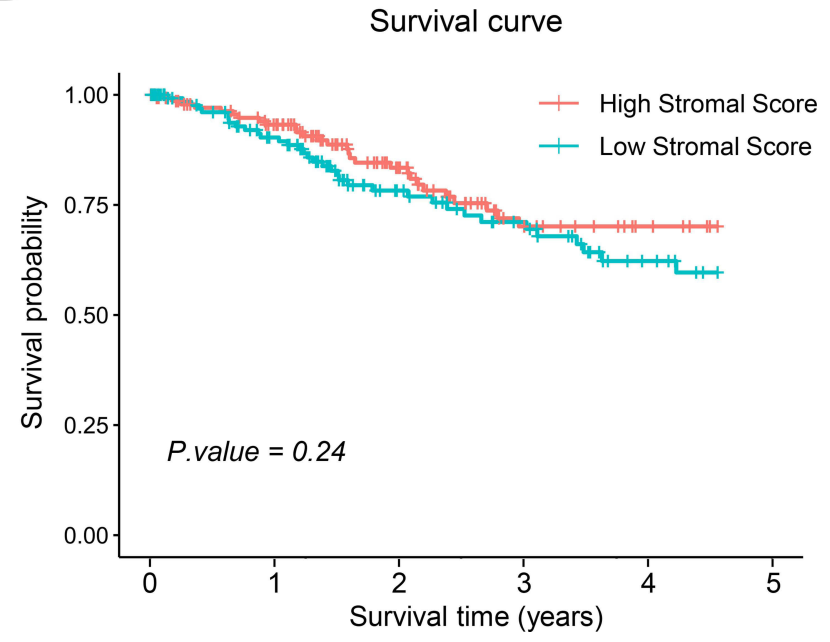

Figure I Prognostic analysis of 29I samples with difference in immune and stromal scores. (A) Prognostic analysis of patients with difference in immune scores. Number of samples in high immune score group was 146. Number of samples in low immune score group was 145. (B) Prognostic analysis of patients with difference in stromal scores. Number of samples in high stromal score group was 146. Number of samples in low stromal score group was 145. 
A

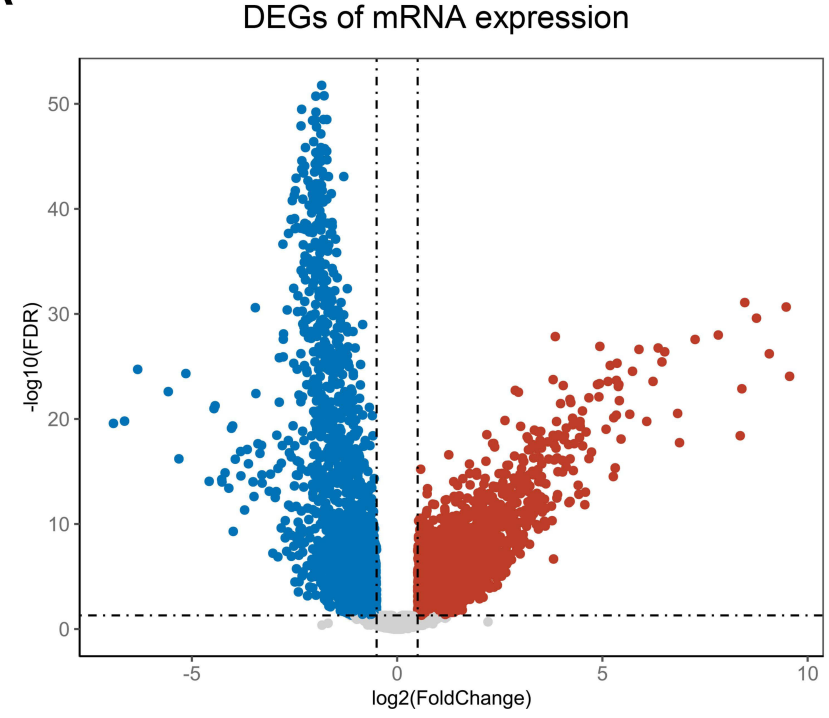

B
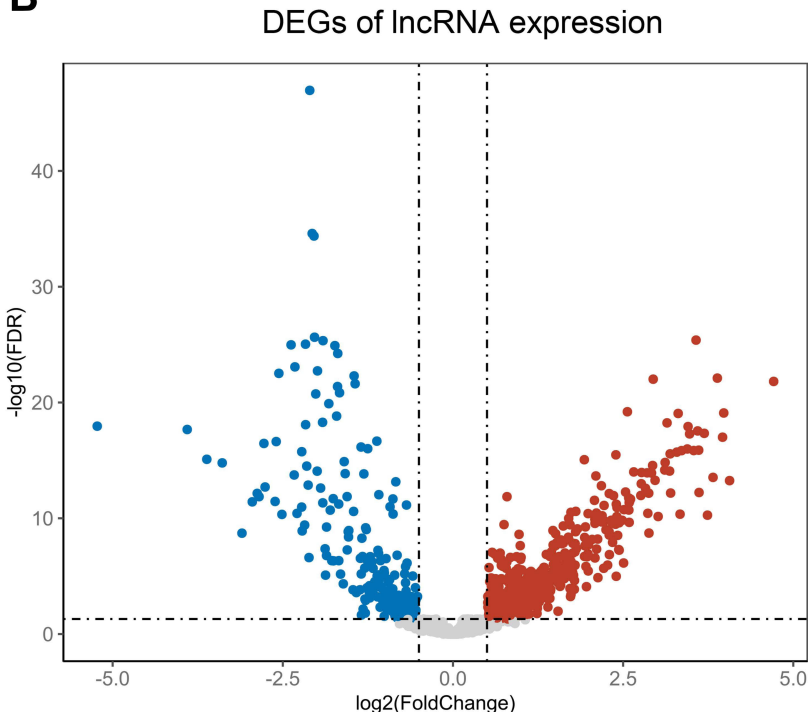

Figure 2 Screening of DEGs and DE-IncRNAs in 291 samples. (A) The volcano maps of DEGs, red dot represents up-regulated mRNAs and blue dot represents downregulated mRNAs in high immune score group. (B) The volcano maps of DE-IncRNAs, red dot represents up-regulated IncRNAs in and blue dot represents down-regulated IncRNAs in high immune score group.

After DEmRNAs and DElncRNAs were screened, the biological meaning of these genes was explored. KEGG pathway enrichment analysis showed that those genes mainly enriched in neuroactive ligand-receptor interaction, cytokine-cytokine receptor interaction and chemokine signaling pathway (Figure 3A). GO pathway enrichment analysis found that those genes mainly enriched in T-cell activation, regulation of lymphocyte activity and leukocyte cell-cell adhesion biological process (Figure 3B). Pathway enrichment results showed that these DEGs mainly associated with chemokine receptor biosynthesis and immune cell activation.

\section{Construction of the Prognosis Model}

To screen lncRNA signatures associated with prognosis of patients with $\mathrm{CC}, 261$ prognosis-associated lncRNAs were identified in training set by univariate Cox analysis. Lasso Cox analysis was processed for further screening
A

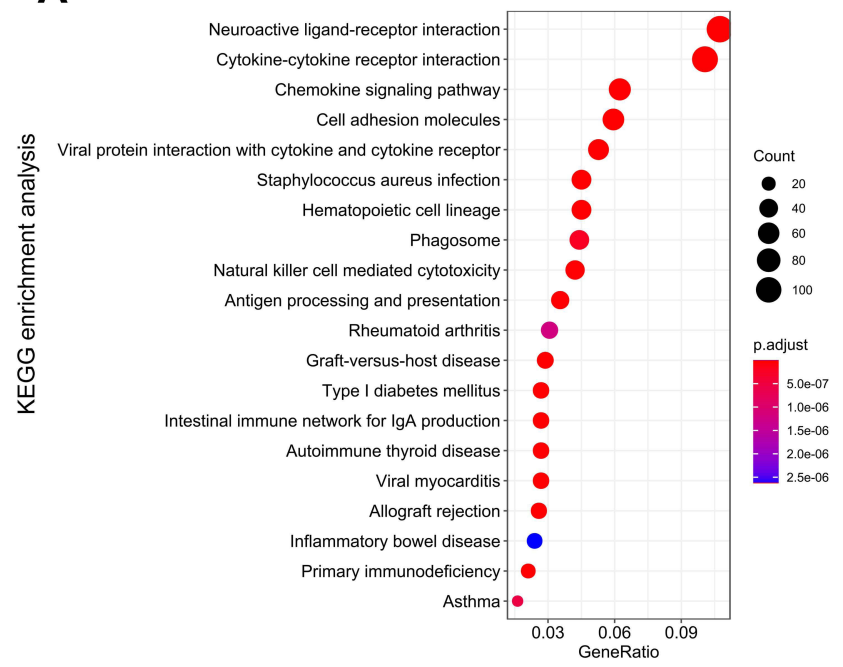

B

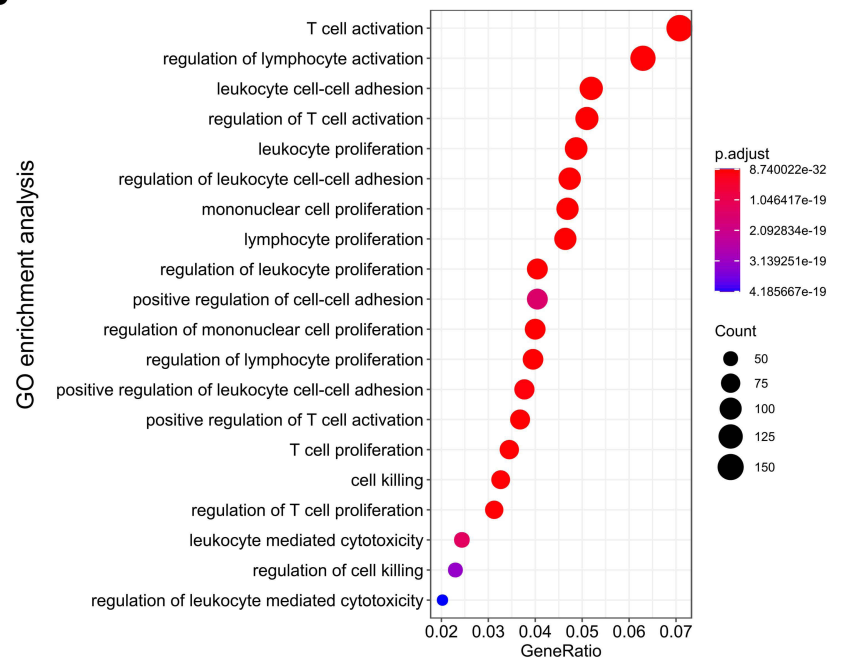

Figure 3 Pathway enrichment analysis. (A) KEGG enrichment analysis. (B) GO enrichment analysis. 
(Figure 4A and B). Finally, multivariate Cox was used to construct a prognosis model (Figure 4C), and the risk score was calculated as:

$$
\begin{aligned}
\text { Riskscore }= & 0.5887611 \times \text { LINC02802 }-0.512341 \\
& \times \text { LINC01281 + 16.745609 × LINC01877 } \\
& -2.857056 \times \text { ZBTB2 }-\mathrm{AS} 1+37.3651331 \\
& \times \text { LINC02480 }+1.5515265 \times \mathrm{WWC} 2-\mathrm{AS} 2 \\
& -2.8238236 \times \mathrm{IFNG}-\mathrm{AS} 1+1.5942727 \\
& \times \text { RBAKDN }
\end{aligned}
$$

After construction of risk model, patients were grouped into two groups by median of risk score. Survival curve and ROC curve were then processed to validate prognosis model. As shown in Figure 4D and F, patients with highrisk scores had significantly worse prognosis outcome than those with low-risk scores in both training cohort and validation cohort. ROC curve shown the prognosis model has a good diagnose efficacy in both training cohort and validation cohort (Figure 4E and G). In conclusion, the lncRNA prognosis model showed a high diagnostic efficacy.

After the prognosis model constructed, survival status and risk scores of $\mathrm{CC}$ patients were illustrated (Figure 5A and $\mathrm{B})$. A heatmap was plotted to visualize the expression of 8-lncRNA signatures in prognosis model (Figure 5C). LINC02802, LINC01877, RBAKDN, LINC02480, WWC2AS2 showed higher expression level in high-risk group while LINC01281, ZBTB20-AS1, IFNG-AS1 expressed higher in low-risk group. We also plotted survival curve of each lncRNA signature to verify the prognosis efficiency of each lncRNA signature. Results indicated that higher expression of LINC02802, LINC01877, RBAKDN, WWC2-AS2 results in significantly longer survival, higher expression of LINC01281, ZBTB20-AS1, IFNG-AS1 results in significantly shorter survival (Figure 6).

\section{GSEA for Functional Annotation of the Immune-Related Risk Signature}

GSEA was performed to investigate pathway activity between low-risk and high-risk groups. Results suggested interferon- $\gamma$ signaling pathway, chemokine signaling pathway, T-cell receptor signaling pathway, CD8 TCR downstream pathway, interleukin 2 family signaling, and interferon signaling were enriched (Figure 7).

\section{Immuno-Correlation Assessment}

To investigate the landscape of immune infiltration in $\mathrm{CC}$, we estimated the immune infiltration level of each immune cell (Figure 8A). The results showed that CD8 T cells were negatively correlative to CD4 T cells and Macrophages M0. Dendritic cells resting was correlative to Mast cells activated (Figure 8B). In addition, higher CD8 T cells, CD4 T cells, and M1 macrophages infiltration were found in low-risk
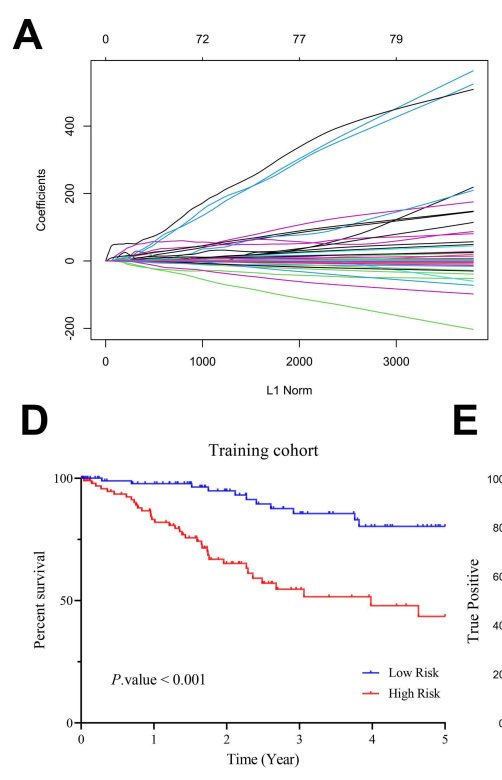
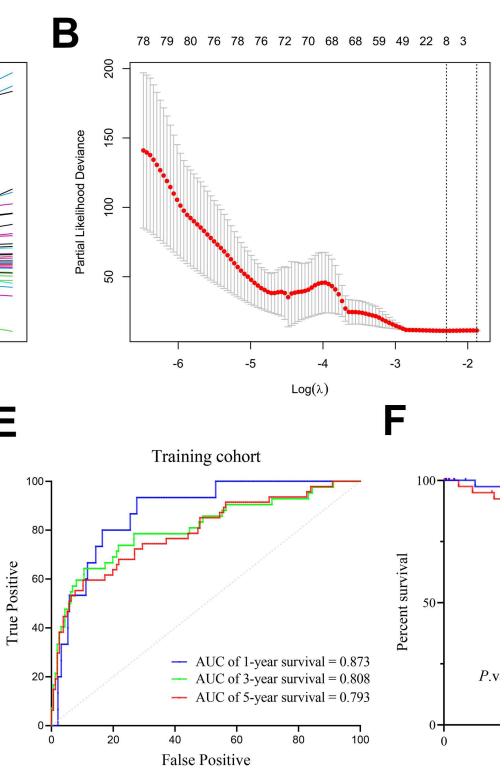

C
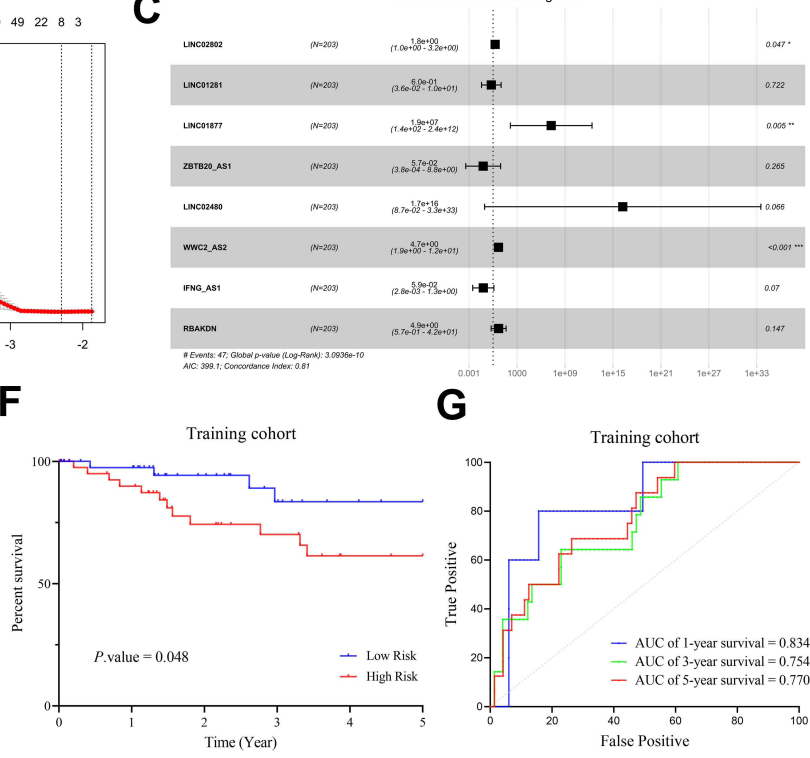

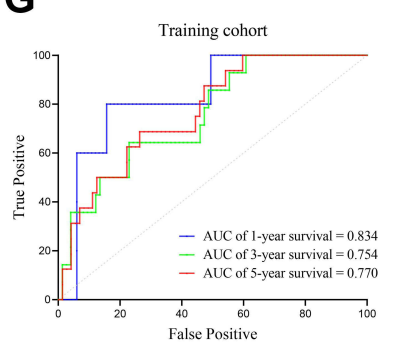

Figure 4 Construction of IncRNA prognosis model. (A and B) The results of lasso regression analysis results. (C) Forest plot shows prognostic IncRNA signatures of CC. (D) OS of patients with high/low-risk score in training cohort. (E) ROC curve of I-year, 3-year and 5-year survival in training cohort. (F) OS of patients with high/low-risk score in validation cohort. (G) ROC curve of I-year, 3-year and 5-year survival in validation cohort. Number of samples in training cohort was 203. Number of samples in validation cohort was 88 . 
A

B

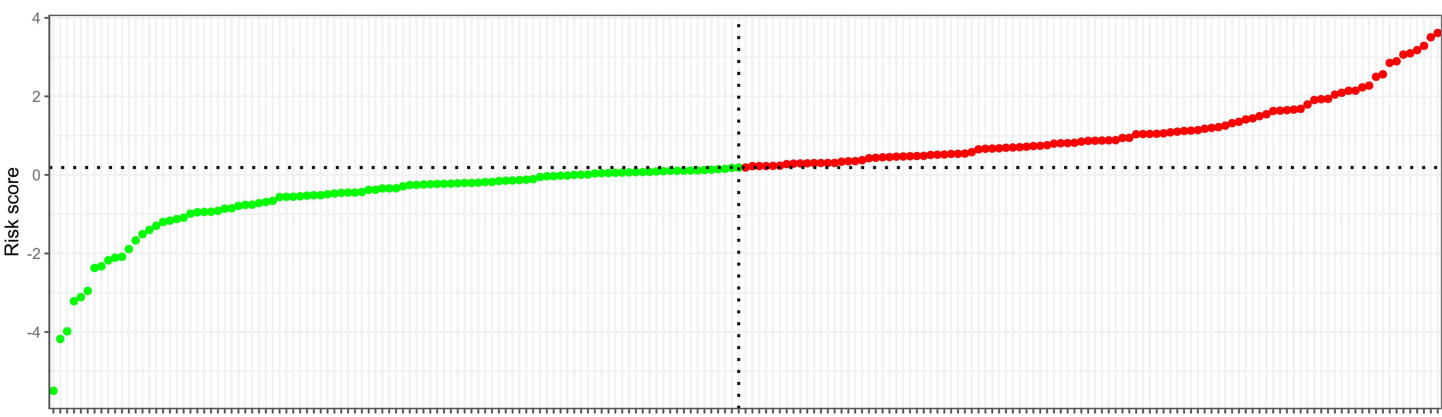

riskgroup

- high

- low
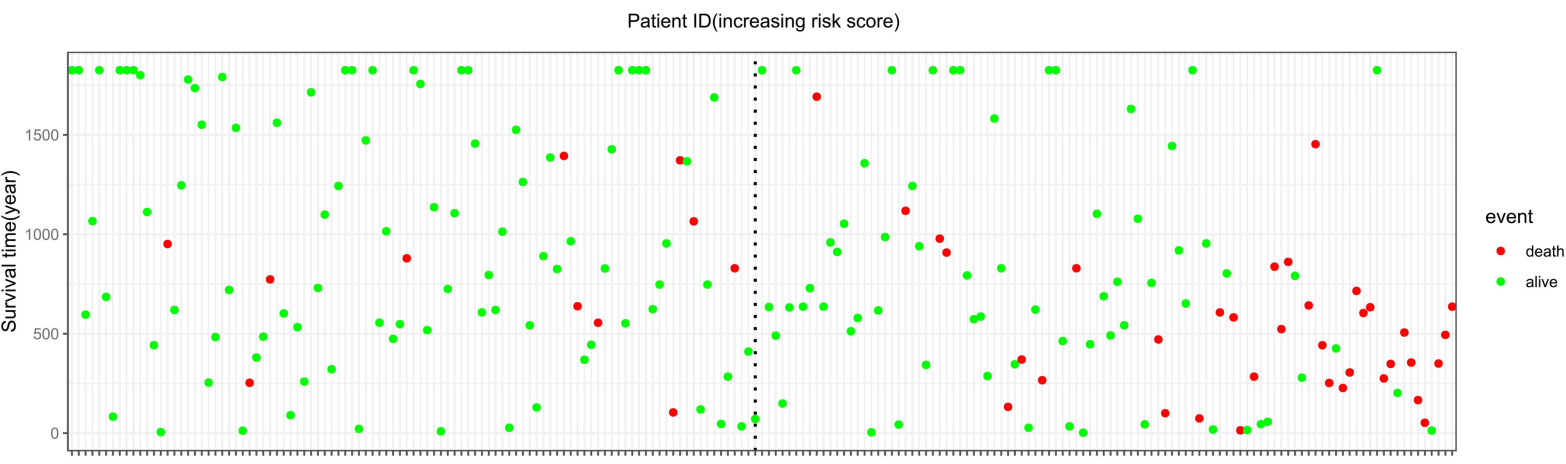

C

Patient ID(increasing risk score)

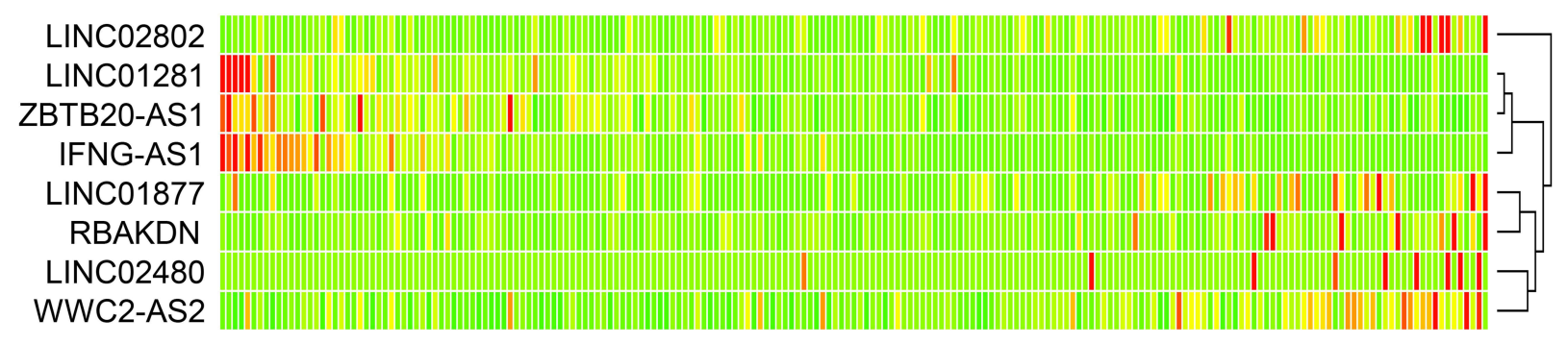

Figure 5 Validation of IncRNA prognosis model in 291 samples. (A) Patients with CC were sorted by increasing risk score. (B) Living status of CC patients. (C) Heatmap of 8 IncRNAs expression profiles of different risk groups. Red color means higher expression while green color represents lower expression. Number of samples in high-risk score group was 146. Number of samples in low-risk score group was 145.

group and higher Dendritic cells and NK cells infiltration were found in high-risk group (Figure 8C). Finally, heatmap was used to visualize the relative abundance of immune cells between two groups (Figure 8D). These findings strongly suggest that the 8-lncRNA signature is associated with prognosis by interfering with immune cell infiltration in CC.

\section{LncRNA Signatures are Correlated with Immune Cell Infiltration}

In our study, the relationship between the 8 immuneassociated lncRNAs and infiltration level of immune cells has also been analyzed respectively. The results showed that $\mathrm{CD} 8 \mathrm{~T}$ cells were positively correlated with BTB20-AS1, LINC01281 and IFNG-AS1.
Dendritic cells were negatively correlated with BTB20AS1, LINC01281, IFNG-AS1 and RBAKDN (Figure 9A). Scatter plot further verified the results (Figure 9B).

\section{Survival Prediction by the 8-IncRNA Classifier is Independent of Clinical Features}

Several analysis methods were used to verify the independence of prognosis model. The univariate Cox analysis revealed that the high-risk group was significantly correlated with shorter survival (Figure 10A). Multivariate Cox regression analyses of these factors indicated that the immune-related lncRNA classifier 

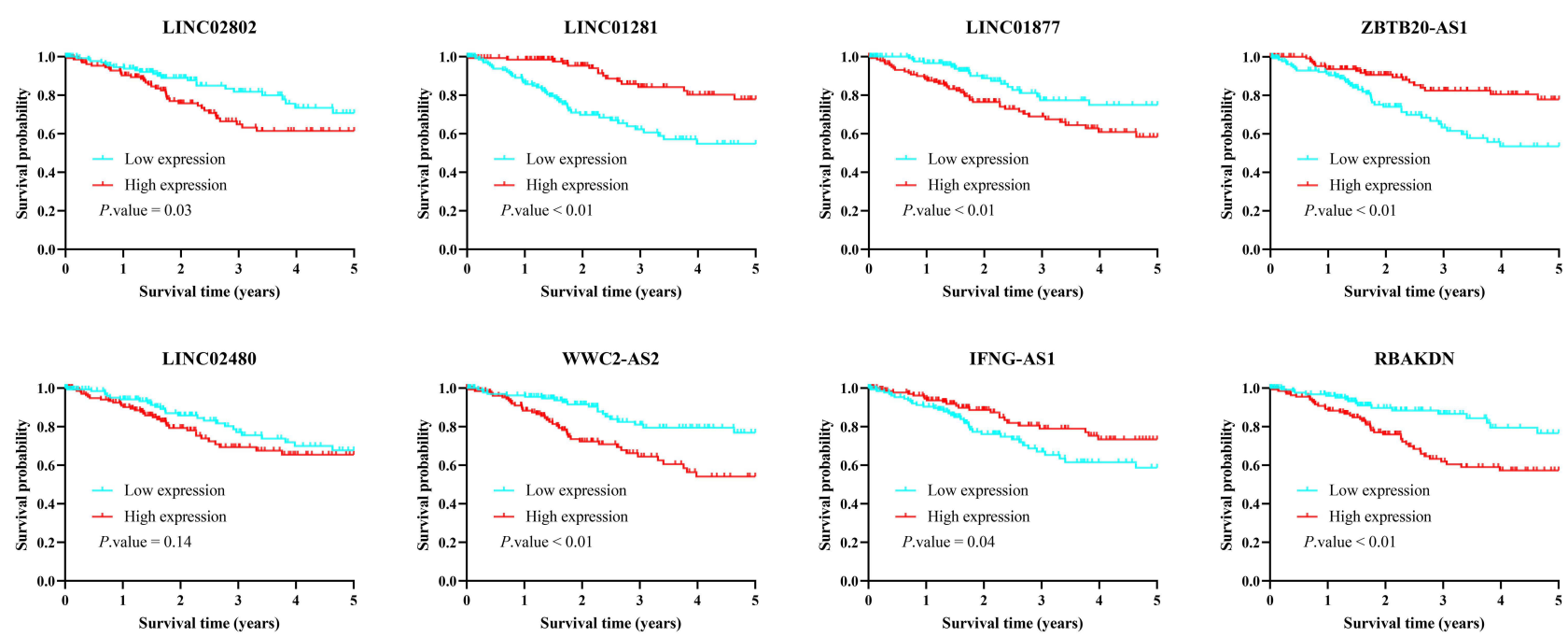

Figure 6 Kaplan-Meier analysis for OS of 29I CC patients based on the expression of 8 IncRNAs.
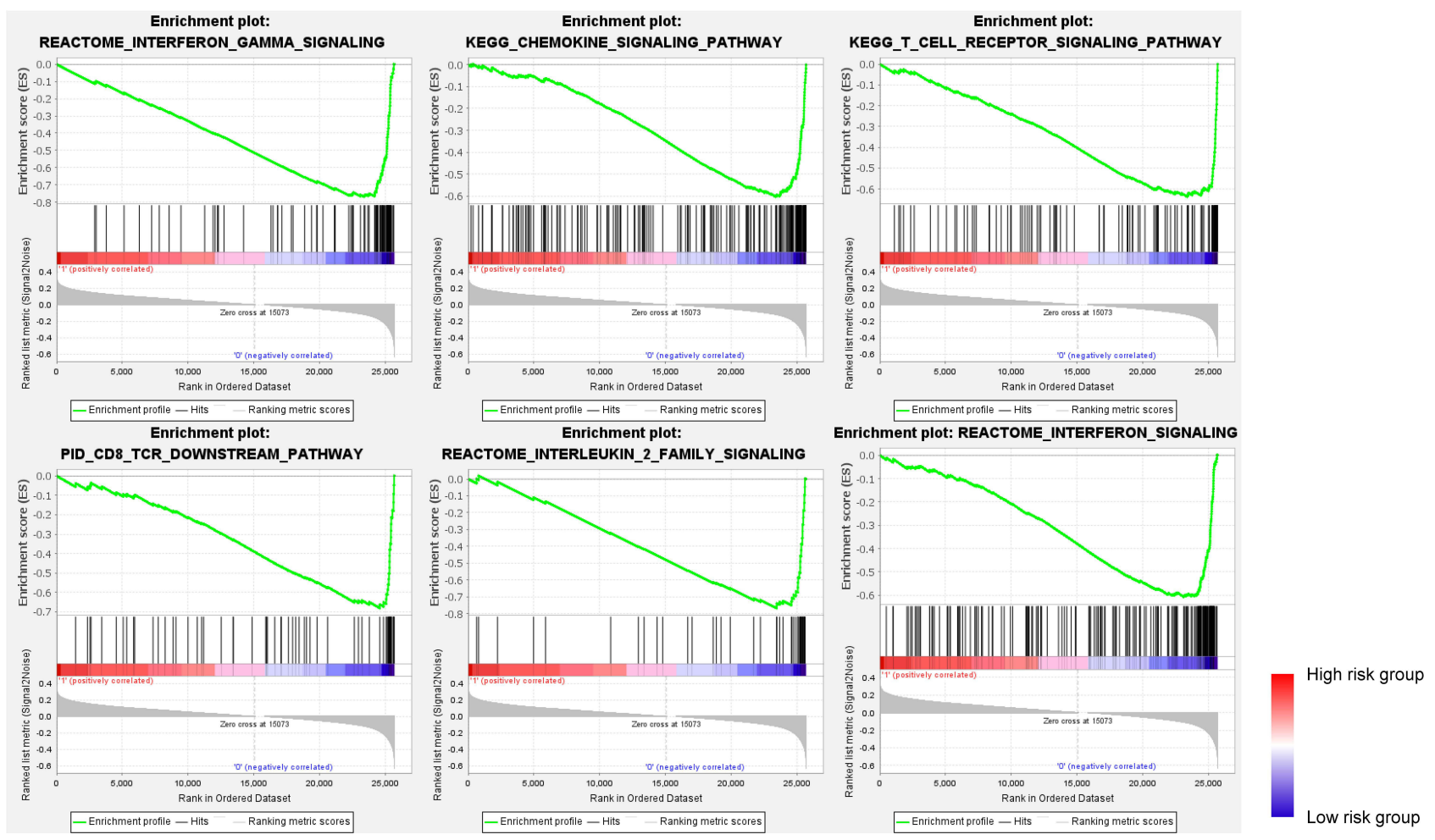

Figure 7 GSEA pathway enrichment analysis of the 8-IncRNA signature.

was a reliable and independent prognostic factor for OS

(Figure 10B). This indicated the prognosis model had a good diagnose efficiency. A nomogram for OS prediction was further built. This nomogram could evaluate the survival rate of patients in 1-year, 3-year and 5-year (Figure 10C).

\section{ZBTB20-ASI and LINCOI28I Promote $T$ Cell Chemotaxis}

To verify the function of the 2 lncRNAs in immune infiltration, T-cell chemotaxis assay was processed. oe-ZBTB20-AS1 and oe-LINC01281 plasmid were transfected into CC cell line (Figure 11A). T-cell chemotaxis assay was used to evaluate 
A

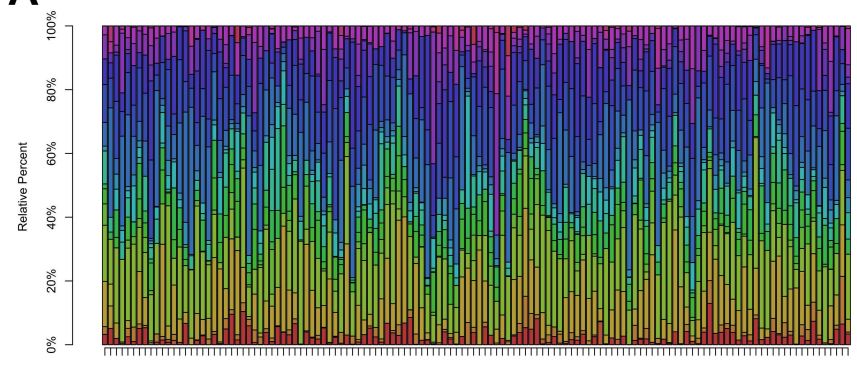

C

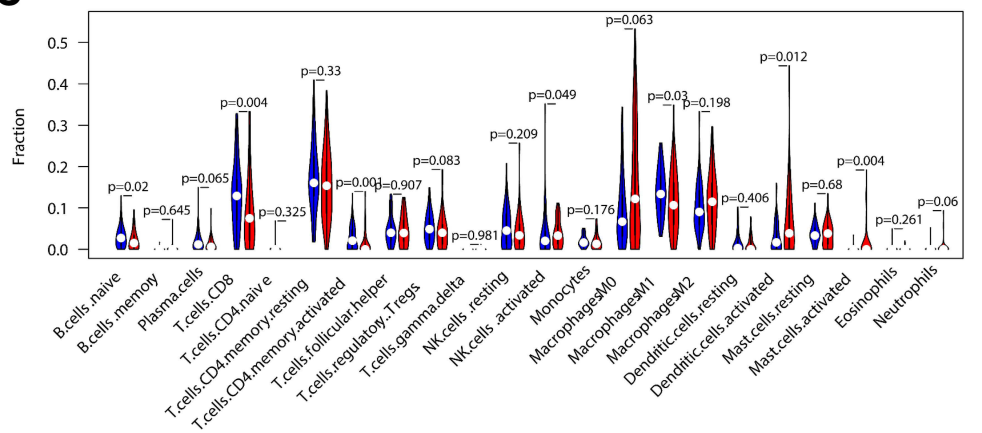

B
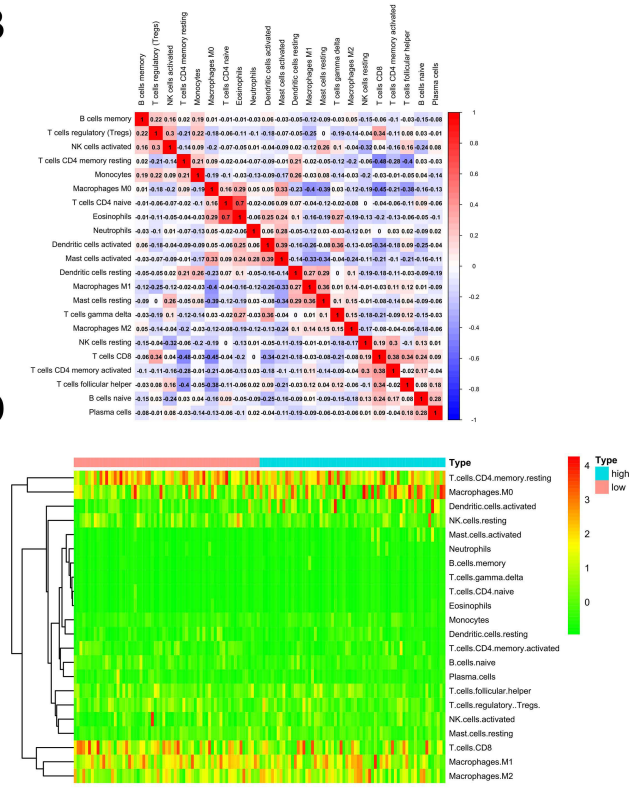

Figure 8 Correlation of the 8-IncRNA signature with immune cell infiltration in 291 samples. (A) Concentration of each immune cell. (B) Correlation analysis of each immune cell. (C) The difference of 22 tumor-infiltrating immune cells among risk groups as defined by the 8-IncRNA signature. Red represents high-risk group while blue represents low-risk group. (D) Heatmap of immune cell relative concentration.

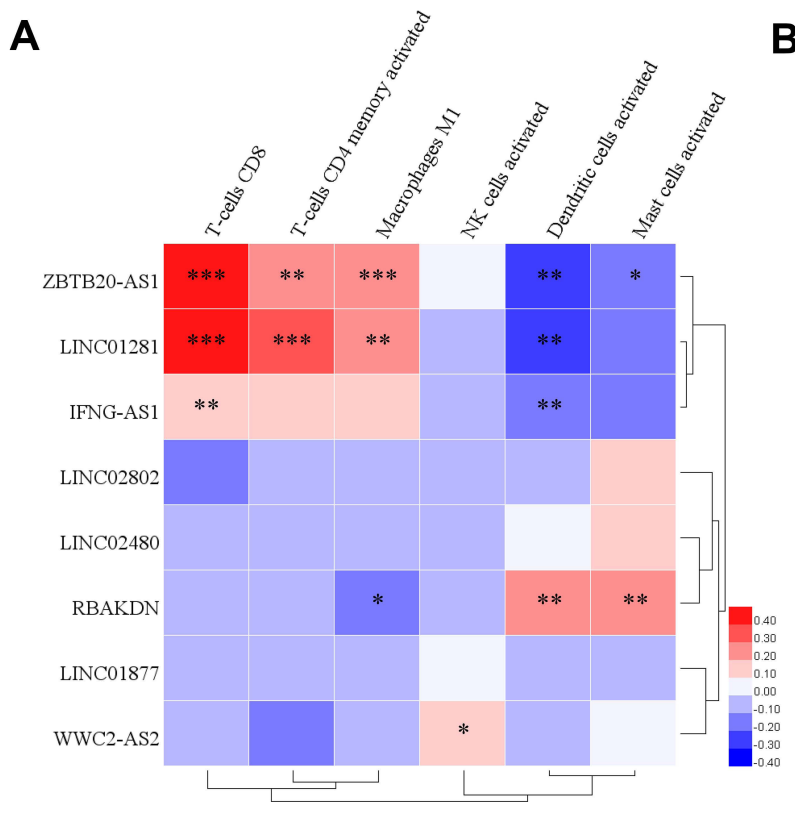

B
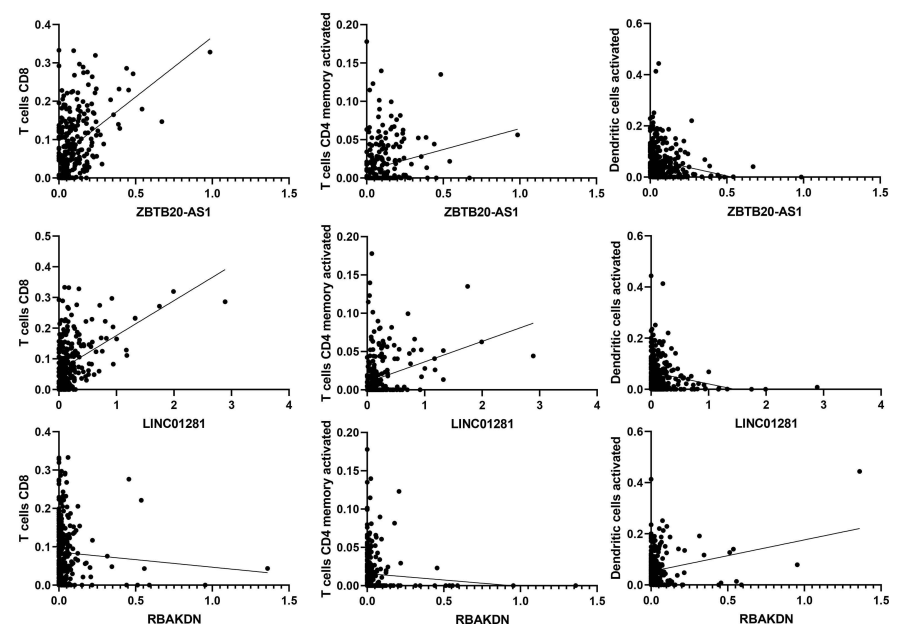

Figure 9 Correlation analysis between IncRNA signatures and immune cell concentration in 29I samples. (A) Heatmap shows the correlation between 8 IncRNA signatures and immune cell concentration. $* P<0.05$, $* * P<0.0$ I, $* * * P<0.00$ I. (B) Scatter plot verifies the correlation between 3 key IncRNA signatures and 3 key immune cells.

the T-cell chemotaxis. The results showed that overexpression of ZBTB20-AS1 and LINC01281 in cancer cell could significantly promote the chemotaxis of $\mathrm{T}$ cells (Figure 11B). Further, qPCR assay was used to identify chemokine and cytokine expression of $\mathrm{CC}$ cells. The results showed that
ZBTB20-AS1 and LINC01281 could up-regulate the expression of CXCL10, CXCL9 and CXCL11 (Figure 11C). These results suggest that $\mathrm{CC}$ cells can release chemokines to attract T cells. ZBTB20-AS1 and LINC01281 can increase the expression level of these chemokines. 


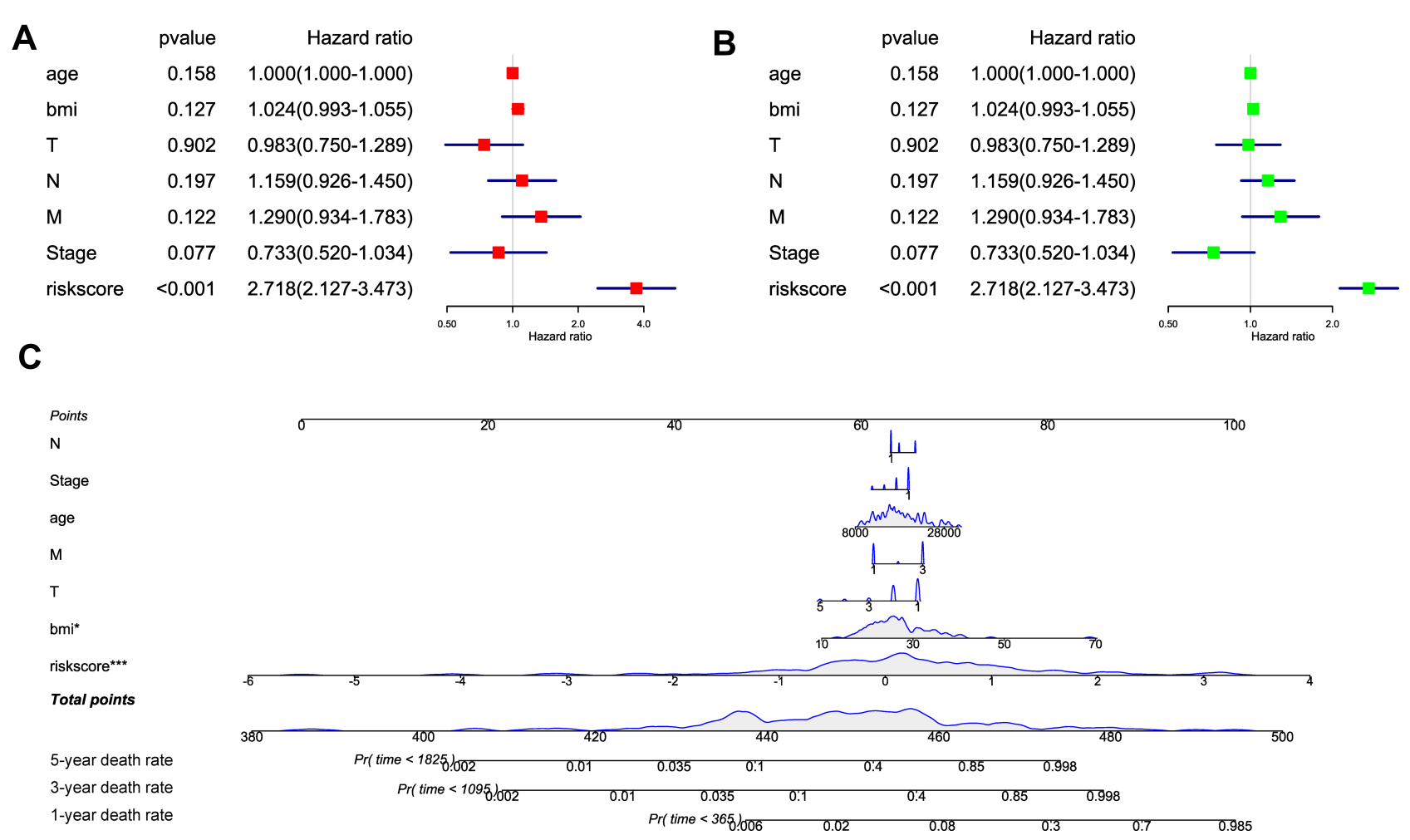

Figure 10 Independent analysis and construction of nomogram. (A) Univariate regression analysis of risk score and other clinical characteristics. (B) Multivariate regression analysis of risk score and other clinical characteristics. (C) Nomogram is constructed to evaluate the I-year, 3-year and 5-year survival rate of $C C$ patients. $* P<0.05$, $* * * P<0.00 \mathrm{I}$.

A

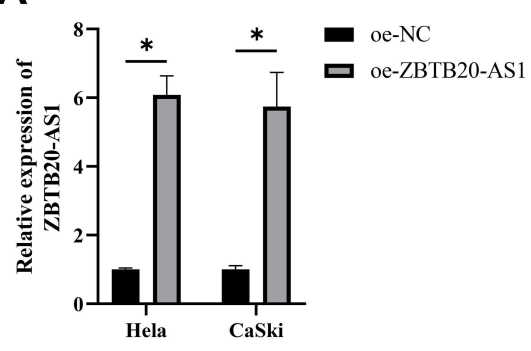

C

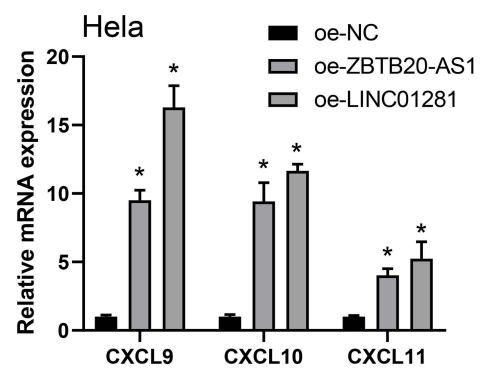

B
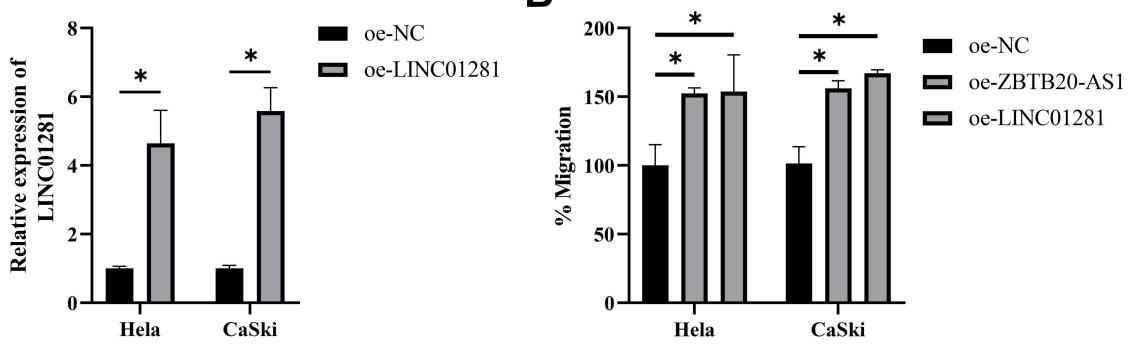

Figure II T cells chemotaxis assay. (A) qPCR assay verified transfection efficiency of two cervical cancer cells. Shown is the mean \pm SD of experiments $(n=3)$, $* P<0.05$. (B) Transwell assay shown T cells chemotaxis. Shown is the mean \pm SD of experiments $(n=3), * P<0.05$. (C) RNA expression of CXCL9, CXCLI0 and CXCLII were detected by RT-qPCR. Shown is the mean \pm SD of experiments $(n=3), * P<0.05$.

\section{Discussion}

Immune landscapes of cancer patients were regarded as good prognosis markers. For example, colorectal carcinoma patients with higher systemic immuneinflammation index (SII) would have shorter survival after surgery. ${ }^{20}$ Although the molecular mechanism of 
immune response remains unclear, immune relative therapies have already shown promising potential., ${ }^{6,7}$ Recent studies found that immune microenvironment played a regulatory role in cancer. ${ }^{21,22}$ In this article, $\mathrm{R}$ package ESTIMATE was used to evaluate the immune infiltration level of CC patients. The results showed that $\mathrm{CC}$ patients with higher immune infiltration could have a better clinical outcome. Similarly, ccRCC patients with higher CD8 $\mathrm{T}$ cell infiltration level were associated with prolonged $\mathrm{OS}^{23}$ These results suggested that immune infiltration could affect the survival rate of $\mathrm{CC}$ patients.

Generally, mRNAs and lncRNAs with high abundance possess significant biological function. We also looked into the function of these DEmRNAs and DElncRNAs which were related to immune infiltration. Pathway enrichment results showed that these DEGs mainly associated with chemokine receptor biosynthesis and immune cell activation. Cytokine interaction and chemokine signaling were two important pathways in immune microenvironment, abnormal expression of cytokines such as TNF- $\alpha$, IL-1, IL-6, IL-8, IL-18 was associated with CC

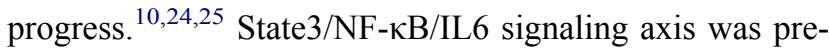
viously proved to promote $\mathrm{CC}$ progression. $^{25,26}$ Chemokines signal proteins could activate various types of immune cells. For example, CXCL chemokine family could affect the progress of $\mathrm{CC} .^{27,28}$ Above studies suggested that immune-related lncRNAs were potentially involved in regulating the remodeling of the immune microenvironment or the activation of immune cells.

LncRNAs are important signatures participate in the regulation of immune system. Some studies found lncRNAs were immune-related prognostic signatures in non-small cell lung cancer, breast cancer and bladder cancer. $^{29-31}$ At the same time, some researchers found lncRNAs could be good prognostic signatures in CC. For example, Zheng and his partners analyzed TCGA and GSE6791 databases and constructed a 4 lncRNA signature which was related to survival rate of $\mathrm{CC}$ patients. ${ }^{32}$ 8-lncRNA signature of $\mathrm{CC}$ has also been identified by integrating DNA methylation, copy number variation and transcriptome data. ${ }^{33}$ In this study, a prognostic model of immune-related lncRNAs was established with multiple regression analysis. We found 8 immune-associated lncRNA signatures: LINC02802, LINC01281, LINC01877, ZBTB20-AS1, LINC02480, WWC2-AS2, IFNG-AS1, RBAKDN. Several lncRNAs were found that they were related to the prognosis of cancer patients
(LINC01281, LINC01877, WWC2-AS2, IFNG AS1, and RBAKDN). ${ }^{10,34-37}$ Among them, IFNG-AS1 played an important role in immune system. LncRNA-IFNG-AS1 have been proved that it could contribute to $\mathrm{T}$ helper type 1 cells response and induce inflammation. ${ }^{38,39}$ It could also promote IFN- $\gamma$ production in nature killer cells and suppress cancer progress. ${ }^{40}$ It is suggested that the 8 lncRNAs could affect the immune response of $\mathrm{CC}$ patients.

The most significant demonstration in our analysis was that we tried to figure out the relationship between lncRNA signature and immune cell infiltration. Pathway activity related to immune response were found different between high and low-risk group with GSEA. Patients with lower risk scores had more active immune responses than those with higher risk scores. In addition, 24 immune-related terms were incorporated to assess the abundance of immune cells in tumor immune microenvironment. CD8 T cells, CD4 T cells, M1 macrophages and dendritic cells infiltration were found to act differently in high- and low-risk groups. CD8 T cells are the main immune cells that kill cancer cells through cytotoxicity effect and CD4 T cells can promote the cytotoxicity effect of immune cells. ${ }^{41-43}$ In this study, CD4 and CD8 T cells infiltration were elevated in low-risk group, this might be conducive to survival rate of $\mathrm{CC}$ patients. In addition, the infiltration level of M1 macrophages in the low-risk group was also significantly increased. Macrophages M1 is a pro-inflammatory cell that can kill tumor cells. ${ }^{42}$ Higher infiltration level of dendritic cells in high-risk group has also been observed. Dendritic cells can suppress the immune response by secreting suppressors such as PD-L1, some studies suggested that dendritic cells could dictate response to immunotherapy and they were good targets for immunotherapy. ${ }^{10,44}$ Interestingly, infiltration of NK cells was found much higher in high-risk group. NK cell played a direct role in tumor killing. ${ }^{45}$ We speculated that the NK cells may play a compensatory role in the tumor microenvironment because the activity of CD4 and CD8 immune cells was inhibited in the high-risk group. Hence, the NK cell infiltration level was higher in the high-risk group.

In our study, the relationship between the 8 immuneassociated lncRNAs and infiltration level of immune cells has also been analyzed, respectively. The results showed that the expression levels of ZBTB20-AS1 and LINC01281 were significantly correlated with the infiltration levels of CD-8 T cells, CD4 T cells and M1 macrophages. We speculated that these two lncRNAs play 
important roles in regulating the level of immune infiltration of immune cells in $\mathrm{CC}$. The results in the present study showed that overexpression of ZBTB20-AS1 and LINC01281 in cancer cell could significantly promote the chemotaxis of $\mathrm{T}$ cells to $\mathrm{CC}$ cells. Over-expression of ZBTB20-AS1 and LINC01281 could also upregulate the expression of CXCL9, CXCL10 and CXCL11 in CC cells. CXCL9 and other chemokines have been found that they could been secreted by cancer cells and attract T-cells. ${ }^{46}$ These studies suggest that CC cells can release chemokines to attract T cells. ZBTB20-AS1 and LINC01281 can increase the expression level of these chemokines.

\section{Conclusion}

In conclusion, we analyzed the difference of immune infiltration in CC patients and established an immunerelated prognosis model based on immune-related lncRNAs. A nomogram comprising an 8-lncRNA signature may help to predict individual odds of death and help clinicians manage patients with breast cancer. Importantly, our IncRNA signature generated and validated in our study might be associated with distinct prognosis outcome of CC patients and immune infiltration levels. Although our results may have potentially significant clinical implications, some issues need to be noted. First, the data in the present study were public database. The accuracy of the risk model should be further examined through clinical samples. In addition, although immune regulation contributes to the development of $\mathrm{CC}$, more complex mechanisms may affect prognosis in colon cancer and require further exploration.

\section{Abbreviations}

ANOVA, analyzed by one-way analysis of variance; AUC, area under curve; BNCC, Beina Chuanglian Biotechnology Institute; CC, cervical cancer; ccRCC, clear cell renal cell carcinoma; DE, differential expression; DEG, differentially expressed gene; FBS, fetal bovine serum; FDR, false discovery rate; GO, gene ontology; GSEA, Gene Set Enrichment Analysis; HPV, human papilloma virus; KEGG, Kyoto Encyclopedia of Genes and Genomes; LASSO, least absolute shrinkage and selection operator; LncRNA, long non-coding RNA; OS, overall survival; PBMC, peripheral blood mononuclear cell; $\mathrm{P} / \mathrm{S}$, Penicillin/streptomycin; ROC, receiver operating characteristic; SII, systemic immune-inflammation index; TCGA, the Cancer Genome Atlas; TIMER, tumor immune estimation resource.

\section{Ethical Approval}

Ethical approval is not applicable for this article. This article does not contain any studies with human or animal subjects.

\section{Funding}

This work is supported by the National Key Research and Development Program of China No. 2016YFC1302900 and the National Natural Science Foundation of China No. 81402364.

\section{Disclosure}

The authors report no conflicts of interest in this work.

\section{References}

1. Landy R, Sasieni PD, Mathews C, et al. Impact of screening on cervical cancer incidence: a population-based case-control study in the United States. Int J Cancer. 2020;147(3):887-896. doi:10.1002/ ijc. 32826

2. Song B, Ding C, Chen W, Sun H, Zhang M, Chen W. Incidence and mortality of cervical cancer in China, 2013. Chin J Cancer Res. 2017;29(6):471-476. doi:10.21147/j.issn.1000-9604.2017.06.01

3. Arbyn M, Weiderpass E, Bruni L, et al. Estimates of incidence and mortality of cervical cancer in 2018: a worldwide analysis. Lancet Glob Health. 2020;8(2):e191-e203. doi:10.1016/S2214-109X(19) 30482-6

4. Liontos M, Kyriazoglou A, Dimitriadis I, Dimopoulos MA, Bamias A. Systemic therapy in cervical cancer: 30 years in review. Crit Rev Oncol Hematol. 2019;137:9-17. doi:10.1016/j. critrevonc.2019.02.009

5. Ventriglia J, Paciolla I, Pisano C, et al. Immunotherapy in ovarian, endometrial and cervical cancer: state of the art and future perspectives. Cancer Treat Rev. 2017;59:109-116. doi:10.1016/j. ctrv.2017.07.008

6. Saglam O, Conejo-Garcia J. PD-1/PD-L1 immune checkpoint inhibitors in advanced cervical cancer. Integr Cancer Sci Ther. 2018;5 (2):10.15761/ICST.1000272. doi:10.15761/ICST.1000272

7. Hu S, Pu D, Xia X, Guo B, Zhang C. CTLA-4 rs5742909 polymorphism and cervical cancer risk: a meta-analysis. Medicine. 2020;99(11):e19433. doi:10.1097/MD.0000000000019433

8. Chung HC, Ros W, Delord JP, et al. Efficacy and safety of pembrolizumab in previously treated advanced cervical cancer: results from the Phase II KEYNOTE-158 Study. J Clin Oncol. 2019;37 (17):1470-1478. doi:10.1200/JCO.18.01265

9. Zhang J, Endres S, Kobold S. Enhancing tumor T cell infiltration to enable cancer immunotherapy. Immunotherapy. 2019;11(3):201-213. doi:10.2217/imt-2018-0111

10. Song YC, Huang HC, Chang CYY, et al. A potential herbal adjuvant combined with a peptide-based vaccine acts against HPV-related tumors through enhancing effector and memory T-cell immune responses. Front Immunol. 2020;11:62. doi:10.3389/ fimmu.2020.00062

11. Pyfferoen L, Brabants E, Everaert C, et al. The transcriptome of lung tumor-infiltrating dendritic cells reveals a tumor-supporting phenotype and a microRNA signature with negative impact on clinical outcome. Oncoimmunology. 2017;6(1):e1253655. doi:10.1080/ 2162402X.2016.1253655

12. Chevrier S, Levine JH, Zanotelli VRT, et al. An immune atlas of clear cell renal cell carcinoma. Cell. 2017;169(4):736-749 e718. doi:10.1016/j.cell.2017.04.016 
13. Liang Y, Lu W, Zhang X, Lu B. Tumor-infiltrating CD8+ and FOXP3+ lymphocytes before and after neoadjuvant chemotherapy in cervical cancer. Diagn Pathol. 2018;13(1):93. doi:10.1186/s13000-018-0770-4

14. Bai X, Wang W, Zhao P, et al. LncRNA CRNDE acts as an oncogene in cervical cancer through sponging miR-183 to regulate CCNB1 expression. Carcinogenesis. 2020;41(1):111-121. doi:10.1093/carcin/bgz166

15. Feng LL, Shen FR, Zhou JH, Chen YG. Expression of the lncRNA ZFAS1 in cervical cancer and its correlation with prognosis and chemosensitivity. Gene. 2019;696:105-112. doi:10.1016/j. gene.2019.01.025

16. Huang D, Chen J, Yang L, et al. NKILA lncRNA promotes tumor immune evasion by sensitizing $\mathrm{T}$ cells to activation-induced cell death. Nat Immunol. 2018;19(10):1112-1125. doi:10.1038/s41590018-0207-y

17. Xu M, Xu X, Pan B, et al. LncRNA SATB2-AS1 inhibits tumor metastasis and affects the tumor immune cell microenvironment in colorectal cancer by regulating SATB2. Mol Cancer. 2019;18(1):135. doi:10.1186/s12943-019-1063-6

18. Kishore M, Cheung KCP, Fu H, et al. Regulatory T cell migration is dependent on glucokinase-mediated glycolysis. Immunity. 2017;47 (5):875-889 e810. doi:10.1016/j.immuni.2017.10.017

19. Juric V, O'Sullivan C, Stefanutti E, et al. MMP-9 inhibition promotes anti-tumor immunity through disruption of biochemical and physical barriers to T-cell trafficking to tumors. PLoS One. 2018;13(11): e0207255. doi:10.1371/journal.pone.0207255

20. Chen JH, Zhai ET, Yuan YJ, et al. Systemic immune-inflammation index for predicting prognosis of colorectal cancer. World $J$ Gastroenterol. 2017;23(34):6261-6272. doi:10.3748/wjg.v23. i34.6261

21. Hong BS, Ryu HS, Kim N, et al. Tumor suppressor miRNA-204-5p regulates growth, metastasis, and immune microenvironment remodeling in breast cancer. Cancer Res. 2019;79(7):1520-1534.

22. McGranahan N, Swanton C. Cancer evolution constrained by the immune microenvironment. Cell. 2017;170(5):825-827. doi:10.1016/j.cell.2017.08.012

23. Zhang $\mathrm{S}$, Zhang $\mathrm{E}$, Long $\mathrm{J}$, et al. Immune infiltration in renal cell carcinoma. Cancer Sci. 2019;110(5):1564-1572. doi:10.1111/ cas. 13996

24. Wang L, Zhao W, Hong J, et al. Association between IL1B gene and cervical cancer susceptibility in Chinese Uygur population: a Case-Control study. Mol Genet Genomic Med. 2019;7(8):e779. doi: $10.1002 / \mathrm{mgg} 3.779$

25. Morgan EL, Macdonald A. Autocrine STAT3 activation in HPV positive cervical cancer through a virus-driven Rac1-NFkappaB-IL -6 signalling axis. PLoS Pathog. 2019;15(6):e1007835. doi:10.1371/ journal.ppat.1007835

26. Su K, Zhao Q, Bian A, Wang C, Cai Y, Zhang Y. A novel positive feedback regulation between long noncoding RNA UICC and IL-6/ STAT3 signaling promotes cervical cancer progression. Am J Cancer Res. 2018;8(7):1176-1189.

27. Zhang W, Wu Q, Wang C, Yang L, Liu P, Ma C. AKIP1 promotes angiogenesis and tumor growth by upregulating $\mathrm{CXC}$-chemokines in cervical cancer cells. Mol Cell Biochem. 2018;448(1-2):311-320. doi:10.1007/s11010-018-3335-7

28. Nagarsheth N, Wicha MS, Zou W. Chemokines in the cancer microenvironment and their relevance in cancer immunotherapy. Nat Rev Immunol. 2017;17(9):559-572. doi:10.1038/nri.2017.49

29. Sun J, Zhang Z, Bao S, et al. Identification of tumor immune infiltration-associated lncRNAs for improving prognosis and immunotherapy response of patients with non-small cell lung cancer. $J$ Immunother Cancer. 2020;8(1):e000110. doi:10.1136/jitc-2019000110
30. Shen Y, Peng X, Shen C. Identification and validation of immune-related lncRNA prognostic signature for breast cancer. Genomics. 2020;112(3):2640-2646. doi:10.1016/j.ygeno.2020.02.015

31. Zhou M, Zhang $\mathrm{Z}$, Bao $\mathrm{S}$, et al. Computational recognition of lncRNA signature of tumor-infiltrating B lymphocytes with potential implications in prognosis and immunotherapy of bladder cancer. Brief Bioinform. 2021;22(3):bbaa047.

32. Zheng J, Cao B, Zhang X, Niu Z, Tong J, Jakiel G. Immune-related four-lncRNA signature for patients with cervical cancer. Biomed Res Int. 2020;2020:3641231. doi:10.1155/2020/3641231

33. Zhong Q, Lu M, Yuan W, et al. Eight-lncRNA signature of cervical cancer were identified by integrating DNA methylation, copy number variation and transcriptome data. J Transl Med. 2021;19(1):58. doi:10.1186/s12967-021-02705-9

34. Wang S, Li MY, Liu Y, et al. The role of microRNA in cisplatin resistance or sensitivity. Expert Opin Ther Targets. 2020;24 (9):885-897. doi:10.1080/14728222.2020.1785431

35. Setiasari DW, Rahmawati G, Sudigyo D, et al. Transcriptome profile of next-generation sequencing data relate to proliferation aberration of nasopharyngeal carcinoma patients in Indonesia. Asian Pac J Cancer Prev. 2020;21(9):2585-2591. doi:10.31557/APJCP.2020.21.9.2585

36. Shen XQ, Wu QM, Yang CH, Yan QD, Cao PJ, Chen FL. Four low expression LncRNAs are associated with prognosis of human lung adenocarcinoma. Clin Lab. 2020;66(10). doi:10.7754/Clin. Lab.2020.200211

37. Huang X, Cai W, Yuan W, Peng S. Identification of key lncRNAs as prognostic prediction models for colorectal cancer based on LASSO. Int J Clin Exp Pathol. 2020;13(4):675-684.

38. Peng H, Liu Y, Tian J, et al. The long noncoding RNA IFNG-AS1 promotes T helper type 1 cells response in patients with hashimoto's thyroiditis. Sci Rep. 2015;5:17702. doi:10.1038/srep17702

39. Padua D, Mahurkar-Joshi S, Law IK, et al. A long noncoding RNA signature for ulcerative colitis identifies IFNG-AS1 as an enhancer of inflammation. Am J Physiol Gastrointest Liver Physiol. 2016;311(3): G446-G457. doi:10.1152/ajpgi.00212.2016

40. Stein N, Berhani O, Schmiedel D, et al. IFNG-AS1 enhances interferon gamma production in human natural killer cells. iScience. 2019;11:466-473. doi:10.1016/j.isci.2018.12.034

41. Farhood B, Najafi M, Mortezaee K. CD8(+) cytotoxic T lymphocytes in cancer immunotherapy: a review. J Cell Physiol. 2019;234 (6):8509-8521. doi:10.1002/jcp.27782

42. Bogen B, Fauskanger M, Haabeth OA, Tveita A. CD4(+) T cells indirectly kill tumor cells via induction of cytotoxic macrophages in mouse models. Cancer Immunol Immunother. 2019;68 (11):1865-1873. doi:10.1007/s00262-019-02374-0

43. Fauskanger M, Haabeth OAW, Skjeldal FM, Bogen B, Tveita AA. Tumor killing by CD4(+) T cells is mediated via induction of inducible nitric oxide synthase-dependent macrophage cytotoxicity. Front Immunol. 2018;9:1684. doi:10.3389/fimmu.2018.01684

44. Peng Q, Qiu X, Zhang Z, et al. PD-L1 on dendritic cells attenuates T cell activation and regulates response to immune checkpoint blockade. Nat Commun. 2020;11(1):4835. doi:10.1038/s41467-020-18570-x

45. Muntasell A, Ochoa MC, Cordeiro L, et al. Targeting NK-cell checkpoints for cancer immunotherapy. Curr Opin Immunol. 2017;45:73-81. doi:10.1016/j.coi.2017.01.003

46. Ma WB, Benavente FC, Santegoets SJAM, et al. EGFR signaling suppresses type 1 cytokine-induced T-cell attracting chemokine secretion in head and neck cancer. PLoS One. 2018;13(9): e0203402. doi:10.1371/journal.pone.0203402 


\section{Publish your work in this journal}

OncoTargets and Therapy is an international, peer-reviewed, open access journal focusing on the pathological basis of all cancers, potential targets for therapy and treatment protocols employed to improve the management of cancer patients. The journal also focuses on the impact of management programs and new therapeutic agents and protocols on patient perspectives such as quality of life, adherence and satisfaction. The manuscript management system is completely online and includes a very quick and fair peer-review system, which is all easy to use. Visit http://www.dovepress.com testimonials.php to read real quotes from published authors. 